$>$ Grâce aux technologies THz (térahertz), il est d'ores et déjà possible de fabriquer des systèmes d'imagerie et il est indéniable que l'arrivée prochaine de sources facilement transportables va révolutionner les techniques de détection à distance. Dans le domaine de la santé, l'apport de cette technologie conduit à un degré de finesse inégalé jusqu'à maintenant dans l'analyse de molécules. Les applications biologiques et médicales, notamment en dentisterie et en dermatologie, sont en cours de développement. Cependant, il ne faut pas pour autant négliger l'étude des répercussions sanitaires des ondes $\mathrm{THz}$; un champ d'investigation est ainsi ouvert et les premiers résultats dans ce domaine sont maintenant disponibles. <

\section{À la frontière onde-lumière}

Que peuvent apporter les techniques térahertz ( $\mathrm{THz}$ ) dans le domaine de la santé ?

Vincent Dabouis, Yves Chancerelle, David Crouzier, Jean-Claude Debouzy

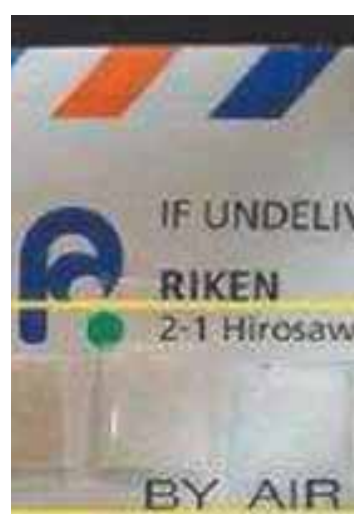

Unité de biophysique cellulaire et moléculaire, Centre de recherche du Service de santé des Armées (CRSSA/BCM),

24, avenue des Maquisdu-Grésivaudan, BP 87 , 38702 La Tronche Cedex, France. vdabouis@crssa.net
Le rayonnement électromagnétique térahertz $(\mathrm{THz}) \mathrm{cou}$ vre un domaine de fréquence allant de $100 \mathrm{GHz}$ à $10 \mathrm{THz}$, c'est-à-dire à la frontière entre les micro-ondes et les infrarouges lointains. De cette position résulte un certain nombre de propriétés optiques ou quasi-optiques (comme la lumière) et de modes de propagation/pénétration proches de ceux des ondes électromagnétiques. Les applications de ce rayonnement dans le domaine biomédical ont vu le jour lorsque les mécanismes de pénétration et de diffusion ont pu être éclaircis [1,2] et utilisés pour différencier des milieux aux propriétés électromagnétiques différentes (conductivité, pénétration) [3]. Plus récemment, le passage des spectroscopies $\mathrm{THz}$ aux technologies impliquant le domaine temporel (THz-TDS) marque une évolution cruciale vers une utilisation croissante de spectromètres miniaturisés ayant de nombreuses applications «de terrain», en particulier dans le domaine médico-légal pour la détection de drogues illicites [4].

Après avoir rappelé les bases physiques des modes d'interaction des ondes $\mathrm{THz}$ avec la matière puis les différentes applications de ces rayonnements dans le domaine de la santé, nous évoquerons de possibles réserves d'ordre sanitaire à l'utilisation régulière de ces nouvelles technologies.

\section{Rappels : les interactions THz-matière}

De manière générale, l'interaction entre une onde électromagnétique et la matière peut se définir soit au niveau corpusculaire (atomique ou moléculaire), soit par la mise en jeu des phénomènes collectifs dans un matériau ou un tissu. Pour qu'il y ait interaction et qu'elle soit enregistrable, il faut qu'il existe un «contraste », c'està-dire que les différentes parties soient plus ou moins réactives (transparentes ou réfléchissantes) afin que l'on puisse les discriminer [5]. Certains objets émettent euxmêmes un rayonnement $\mathrm{THz}$ à température ambiante, dont l'intensité est proportionnelle à leur absorption. Le milieu environnant doit, bien entendu, être transparent pour que le rayonnement issu de l'objet atteigne le détecteur. II convient donc dans un premier temps de décrire l'interaction entre une onde électromagnétique $\mathrm{THz}$ et la matière, afin de comprendre pourquoi un matériau est plus ou moins opaque, réfléchissant ou émissif dans ce domaine spectral. Ce dernier s'étend typiquement entre 0,1 et $10 \mathrm{THz}$ correspondant à des longueurs d'onde de $30 \mu \mathrm{m}$ à $3 \mathrm{~mm}$ (Figure 1). L'énergie des photons (entre 0,4 et $40 \mathrm{meV}$ ) est trop faible pour interagir avec les transitions entre états électroniques des atomes ou molécules $(\sim \mathrm{leV})$ ou avec les énergies de vibration 


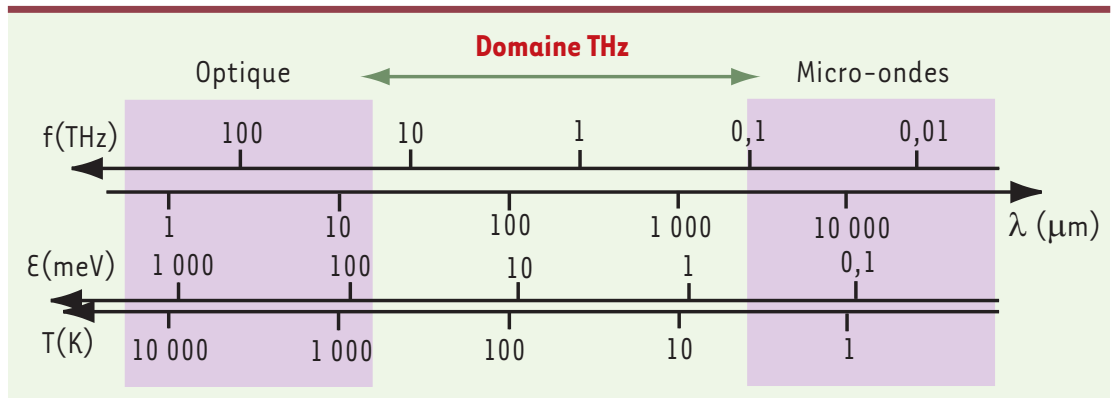

Figure 1. Le domaine $\mathrm{THz}$ en fonction de l'échelle de fréquence, de longueur d'onde, d'énergie (d'après [5]).

moléculaire $(40 \sim 100 \mathrm{meV})$. Ces photons ne peuvent exciter que des résonances de faible énergie au sein de la matière. L'interaction onde $\mathrm{THz}$-molécule sera intense lorsque la molécule portera un fort moment dipolaire comme c'est le cas des molécules d'eau. Pour la matière solide, plusieurs modes d'excitation existent: vibrations collectives du cristal (phonons), excitation de porteurs libres (métaux et semi-conducteurs) etc. De manière pratique, chaque fois qu'une résonance est susceptible d'être mise en jeu dans ce domaine, l'onde est absorbée et le matériau apparaît opaque en imagerie. De plus, si ces résonances sont assez étroites du point de vue spectral, la réponse THz porte la signature spécifique du matériau. Ainsi pourra-t-on dresser l'image des objets dans le domaine $\mathrm{THz}$ en s'appuyant sur leurs propriétés de transparence; de plus, l'analyse de la réponse spectrale de l'objet permettra de connaître sa composition chimique. Pour les applications en imagerie, l'indice de réfraction des matériaux joue aussi un rôle important. Ces deux paramètres sont interdépendants, l'indice de réfraction présentant une forte variation au voisinage du pic d'absorption.

\section{Applications de la spectroscopie térahertz dans le domaine temporel}

La spectroscopie THz-TDS (THz-TDS terahertz time-domain spectroscopy) est basée sur l'utilisation d'impulsions électromagnétiques ultrabrèves d'une durée de l'ordre de la picoseconde permettant d'acquérir un spectre associé couvrant le domaine THz. Ces impulsions permettent de caractériser des matériaux ou dispositifs en transmission et/ou en réflexion. Le principe de la caractérisation en transmission est donné sur la Figure 2. À partir des enregistrements temporels des impulsions $\mathrm{THz}$ obtenus avec puis sans le dispositif à caractériser, on obtient, par transformée de Fourier, les spectres de ces impulsions $\mathrm{THz}$. Leur rapport conduit au coefficient de transmission complexe de l'échantillon, lequel est lui-même fonction de l'indice de réfraction et du coefficient d'absorption du matériau et de son épaisseur [6]. La génération et la mesure des impulsions $\mathrm{THz}$ sont réalisées à partir d'impulsions optiques ultrabrèves délivrées par des lasers femtosecondes.

La technique THz-TDS constitue l'un des rares moyens d'étude de la stabilité de molécules liées entre elles par des interactions faibles, par exemple des liaisons hydrogènes. De nombreuses molécules biologiques telles que les protéines, les polypeptides et I'ADN sont stabilisées par de telles liaisons [7, 8]. L'analyse THz-TDS de ces composés fournit donc d'importantes informations sur les dynamiques conformationnelles de ces biomolécules et peut être utilisée comme moyen d'identification. Dans l'exemple de la myoglobine, Ueno et al. [9] ont montré qu'il était possible de mettre en évidence, par un profil spectral THz, l'existence de modes rotationnels et vibrationnels propres qui gouvernent les propriétés de cette molécule.

Dans un autre domaine, certains médicaments peuvent exister sous plusieurs formes solides ayant des structures cristallines, et donc des propriétés physicochimiques différentes. Elles sont appelées polymorphes et se forment durant la phase de cristallisation. Leur production peut être contrôlée lors de la synthèse et surtout, elles peuvent évoluer lors d'un stockage incorrect ou lors de la

Figure 2. Principe de la caractérisation d'un échantillon par spectroscopie $\mathrm{THz}$ dans le domaine temporel. TF : transformée de Fourier (d'après [6]). 


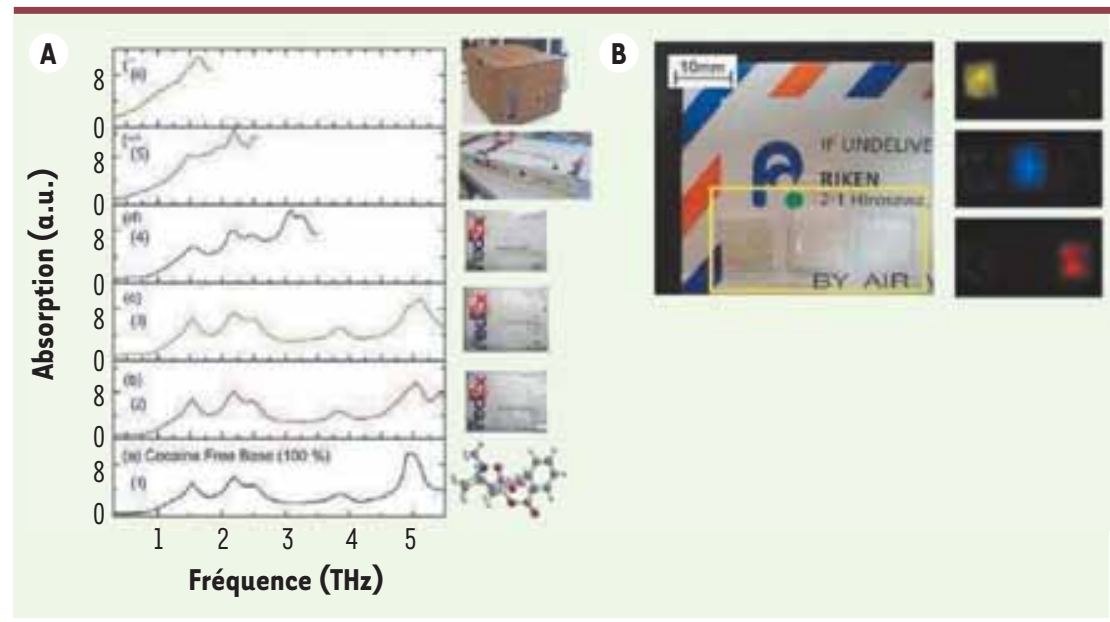

Figure 3. A. Spectres d'un comprimé de cocaine base pure ( 1 ) enveloppé dans différents types d'emballage (de 2 à 6). B. Image montrant 3 sachets en polyéthyène contenant de gauche à droite du NMDA, de l'aspirine et de la metamphétamine. Ces sachets étaient placés dans l'enveloppe ci jointe. À droite, les trois échantillons repérés par la technologie $\mathrm{THz}$ (d'après [4]).

fabrication de comprimés. Á ce titre, différentes formes spectrales de la carbamazépine, médicament utilisé dans le traitement des épilepsies et autres désordres neurologiques, ont été identifiées par analyse $\mathrm{THz}$ montrant que cette technique est adaptée aux études de polymorphisme structural $[10,11]$.

La spectroscopie $\mathrm{THz}$ est aussi particulièrement utile pour l'analyse de structures énantiomériques, en particulier lors d'études de biomolécules cristallisables [10]. L'étude $\mathrm{THz}$ a ainsi révélé l'évolution totalement différente des spectres du tryptophane $D$ ou $L$ après ajout d'un fluor. Cette substitution est difficilement mise en évidence par d'autres techniques.

De nombreuses autres applications de cette technique existent. Citons, pêle-mêle :

- l'étude en biologie structurale de la dynamique de solvatation de composés hydratés $[1,12,13]$.

- Dans le domaine de l'écotoxicologie, la détection de radicaux NO dans des milieux réactifs tels qu'ils sont utilisés en combustion [14] ou pour détecter et quantifier des polluants en phase gazeuse [15].

- dans le domaine de l'industrie pharmaceutique, une meilleure compréhension de la qualité et de la performance des mécanismes de délivrance des principes actifs des médicaments. Le rayonnement $\mathrm{THz}$ pénètre facilement à travers le plastique ou les polymères utilisés dans l'industrie pharmaceutique et permet aussi l'analyse des principes actifs de la plupart des médicaments [10].

\section{Applications de l'imagerie térahertz pulsée (ITP)}

\section{Applications pharmaceutiques}

La radiation $\mathrm{THz}$ peut être utilisée en tant que sonde à l'intérieur même de l'objet d'étude et l'exemple de l'évaluation des comprimés à libération prolongée (CLP) en est une bonne illustration [16]. La plupart des excipients utilisés en galénique forment l'enrobage et sont transparents à la lumière $\mathrm{THz}$. Le principe actif et les différents composants qui l'entourent apportent un contraste image important dû aux différents indices de réfraction des différentes couches du comprimé. Lorsqu'une radiation cohérente ultracourte térahertz est dirigée sur sa surface (système multicouches), une partie de l'impulsion est réfléchie à travers les différentes surfaces de l'enrobage et donne alors une image contrastée. Le rapport des radiations réfléchies sur les radiations transmises permet d'avoir directement accès aux propriétés diélectriques des différents composés. Plus l'onde THz pénètre profondément dans le comprimé, plus il y a de réflexions et donc de signal. Ainsi, l'épaisseur des différentes couches peut être déterminée de manière non destructive par le calcul de temps de vol des impulsions et les index de réfraction des différents revêtements du comprimé.

L'ITP a été aussi utilisée pour la compréhension de la fabrication et particulièrement de l'encapsulation des CLP. Cette technique apporte bien plus d'informations quantitatives sur les propriétés physico-chimiques du revêtement (épaisseur de l'encapsulation, reproductibilité, distribution et uniformité de l'enrobage) que les techniques classiques [16].

Dans l'avenir, un des défis majeurs sera d'incorporer dans les analyses de données la part prise par les effets dispersifs et diffractants de l'analyte vis-à-vis du rayonnement $\mathrm{THz}$ afin de mieux caractériser la structure interne profonde des composés analysés [5].

\section{Applications à la détection}

\section{des drogues ou d'agents biologiques}

L'utilisation des propriétés de transparence des tissus peut permettre de développer des caméras susceptibles de voir à travers les vêtements ou les murs, et dans le domaine de la santé, l'utilisation de techniques d'imagerie multi/hyper spectrale dans la gamme THz ouvre la possibilité d'une détection à distance d'agents biologiques et chimiques cachés dans du courrier ou des bagages, qui devient possible grâce au développement récent de systèmes compacts utilisant la technologie $\mathrm{THz}$ laser femtoseconde PCL (quantum cascade laser) [17].

Un certain nombre de drogues illicites a été étudié, en particulier les amphétamines, la cocaïne base (crack), 

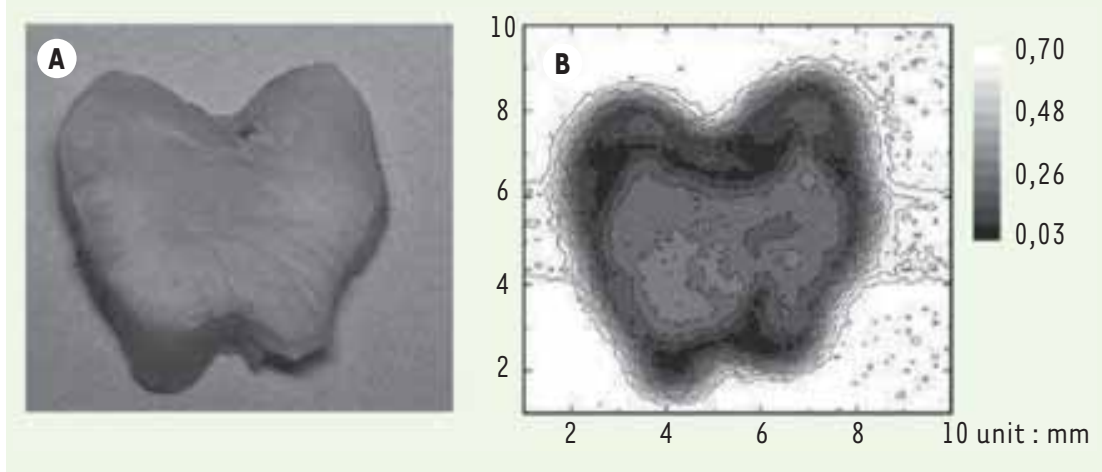

Figure 4. Image optique (A) et image par transmission d'amplitude $\mathrm{THz}_{\mathrm{z}}$ (B) d'un échantillon de dent (d'après [21]).

ou son dérivé hydrochloride (coke), le MDMA ou extasy, la morphine ou l'héroïne (Figure 3). L'avantage de la THz-TDS est sa capacité à détecter ces produits même s'ils ont été délibérément dissimulés du fait de la bonne transmission du rayonnement $\mathrm{THz}$ à travers les dispositifs qui les contiennent [4]. Par ailleurs, des études sur la détection d'agents biologiques ont été publiées par Choi et al. Les auteurs ont effectué des mesures de transmission sur l'enveloppe membranaire de $B$. cereus simulant l'action de B. anthracis [18]. Les signatures spectrales hautement spécifiques ont été enregistrées et prouvent que cette technique a un avenir certain pour la détection de ce type d'agression.

\section{Applications médicales}

Deux propriétés des radiations $\mathrm{THz}$ gouvernent leur utilisation in vivo: - compte tenu de la faible épaisseur de pénétration de l’onde, seules la peau et les dents sont accessibles à l'analyse $\mathrm{THz}$,

- l'atténuation $\mathrm{THz}$ est sensible au contenu en eau et donc peut intéresser les études relatives à l'hydratation des tissus.

La peau est l'organe le plus accessible de l'organisme; elle l'est donc à l'imagerie $\mathrm{THz}$. Sa structure stratifiée en couches, principalement l'épiderme, le derme et l'hypoderme, est intéressante pour l'imagerie par réflexion du fait des interfaces entre les différentes strates. II est effectivement possible de différencier ces couches de la peau humaine [19] et de mettre en évidence non seulement une pathologie (par ex. le psoriasis) mais aussi de suivre les modifications structurales induites par un traitement, ou de distinguer la peau normale d'un carcinome basocellulaire [1]. Cette approche moins coûteuse que l'imagerie par résonance magnétique ne nécessite pas de contact physique comme avec les techniques ultrasoniques. Il est également possible de suivre l'évolution (hémostase, granulation et cicatrisation) de plaies d'origine traumatique ou thermique au cours du traitement qui peut alors être adapté. Par ailleurs, l'eau contenue dans le stratum corneum peut affecter les propriétés physiques de la peau, à savoir sa fonction de barrière. L'étude par THz de l'hydratation de la peau est donc un moyen d'appréciation de l'efficacité d'un cosmétique [20].

Dans un proche avenir, on pourra aussi distinguer les tissus ischémiques des tissus sains, ce qui a un intérêt diagnostic, pronostique et thérapeutique. II a été aussi démontré que la TPI pouvait avoir un rôle intéressant dans le suivi de tumeurs solides superficielles non métastasées [19]. Au même titre que la peau, les dents sont accessibles à l'ITP (Figure 4); cette technique peut détecter, de manière plus précoce que la radio- graphie conventionnelle, la présence d'une carie dans l'émail et donc la mise en place plus précoce et donc plus efficace d'un traitement [21,22].

Des résultats prometteurs ont été publiés concernant le cancer du poumon [23]. L'ITP a permis d'identifier de manière précise la marge des cellules tumorales et ainsi de définir pour le chirurgien les zones exactes à exciser. Les fréquentes sous-estimations de la taille des tumeurs peuvent ainsi être limitées, évitant une reprise chirurgicale secondaire. De même, chez un patient atteint de cancer du foie, la partie tumorale a été identifiée par une image $\mathrm{THz}$ à haute résolution spatiale [7].

Dans le domaine de la recherche fondamentale, citons enfin le criblage de gènes par onde $\mathrm{THz}$ [24] et plus récemment, l'espoir que suscite l'analyse automatique de l'ADN par THz [5]. En effet, suivant le degré d'hybridation de l'ADN, sa réponse aux champs THz est différente et mesurable.

\section{Effets sanitaires du rayonnement $\mathrm{THz}$}

Même si, à la différence des rayons $X$ ou gamma, le rayonnement $\mathrm{THz}$ susceptible d'être utilisé pour l'imagerie ne semble pas présenter de caractère de dangerosité connu pour l'être humain [25], les études de toxicité des rayonnements $\mathrm{THz}$ sont encore rares. Doria et al. [26] se sont intéressés aux interactions entre les émissions $\mathrm{THz}$ et les systèmes biologiques, thème du projet européen $\mathrm{THz}-$ Bridge Project. Les auteurs ont utilisé un système de membranes cellulaires artificielles (liposomes) pour l'étude de la perméabilité membranaire et des lymphocytes humains pour identifier des effets génotoxiques potentiels. Si une modification de la perméabilité membranaire des liposomes a été constatée, elle ne s'accompagne d'aucune activité génotoxique dans les tests classiques (test comète et micronoyaux). Très récemment, Korenstein et al. [27] ont montré que l'effet d'une radiation continue de $0,1 \mathrm{THz}$ $\left(0,031 \mathrm{~mW} / \mathrm{cm}^{2}\right)$ sur des lymphocytes humains en phase de division est réel. Les chromosomes 11 et 17 ont été les plus vulnérables: on détecte une augmentation de $30 \%$ d'aneuploïdie ainsi que des changements de type 
asynchrone des réplications centromériques. Une instabilité génomique induite par un rayonnement $\mathrm{THz}$ faible puissance laisse supposer une augmentation potentielle du risque de cancers.

\section{Conclusion}

Les technologies THz bénéficient depuis ces dernières d'années d'un engouement scientifique certain comme en témoigne le nombre de publications en croissance exponentielle $[28,29]$. Il est d'ores et déjà possible de trouver des systèmes d'imagerie capables de détection à travers les vêtements et il est indéniable que l'arrivée prochaine de sources $\mathrm{THz}$ compactes, facilement transportables et abordables financièrement, risque de révolutionner les techniques de détection à distance. L'apport de la TDS THz dans l'analyse de molécules permet de mesurer des modes vibrationnels à un degré de finesse sans précédent et de réaliser des empreintes spectrales de plus en plus précises et donc une modélisation numérique plus proche de la réalité. Les applications biologiques et médicales, notamment en dentisterie et en dermatologie, sont pour l'heure en cours de mise au point ou de développement. Cependant, il ne faut pas pour autant négliger l'étude des répercussions sanitaires des ondes $\mathrm{THz}$; un champ d'investigation est ainsi ouvert et les études dans ce domaine sont plus que jamais nécessaires. $\diamond$

\section{SUMMARY}

What's new in biomedical applications

for Terahertz (THz) technology

Terahertz technologies have recently been applied to develop high resolution imaging. Since practical portable systems can be designed, the possibilty has emerged to easily screen for biohazards and concealed objects, a procedure which usually requires remote analysis. Applications of $\mathrm{THz}$ are also envisaged in the medical field, because this technology offers a degree of accuracy never reached before in molecule analysis. Skin abnormalities and dental health care are two promising targets of $\mathrm{THz}$ applications. Nevertheless, potential hazards and health effects of $\mathrm{THz}$ exposure should be monitored carefully, particularly since some data suggest induction of genomic instability. $\diamond$

\section{REMERCIEMENTS}

Nous tenons à remercier le professeur Guy Lallement pour sa relecture constructive du document.

\section{CONFLIT D'INTÉRÊTS}

Les auteurs déclarent n'avoir aucun conflit d'intérêts concernant les données publiées dans cet article.

\section{RéFÉRENCES}

1. Wallace VP, Taday PF, Fitzgerald AJ, et al. Terahertz pulsed imaging and spectroscopy for biomedical and pharmaceutical applications. Faraday Discuss 2004 ; $126: 255-63 / 303-11$.

2. Humphreys K, Loughran JP, Gradziel M, et al. Medical applications of terahertz imaging: a review of current technology and potential applications in biomedical engineering. Conf Proc IદEદ Eng Med Biol Soc 2004 ; 2 : 1302-5.

3. Smye SW, Chamberlain JM, Fitzgerald AJ, Berry $\varepsilon$. The interaction between Terahertz radiation and biological tissue. Phys Med Biol $2001 ; 46$ : R101-12.
4. Davies AG, Linfield EH, Johnston MB. Terahertz spectroscopy of explosives and drugs. Materials Today $2008 ; 11: 18-26$.

5. Coutaz JL, Garet F, Gaborit G, Duvillardet L. Potentialités des ondes térahertz en imagerie. CR Physique 2007 ; 9 : 1-10.

6. Duvillaret L, Garet F, Coutaz JL. A reliable method for extraction of material parameters in $\mathrm{THz}$ time-domain spectroscopy. IદદEJ 1996 ; $2: 739-46$

7. Nishizawa J, Sasaki T, Tanno T. Coherent terahertz-wave generation from semiconductors and its applications in biological sciences. J Phys Chem Sol $2008 ; 69: 693-701$.

8. Laman N, Harsha SS, Grischkowsky D, Melinger JS. High-resolution waveguide $\mathrm{THz}$ spectroscopy of biological molecules. Biophys J 2008; $94: 1010-20$.

9. Ueno Y, Ajito K. Analytical terahertz spectroscopy. Anal Sci 2008 ; $24: 185-92$.

10. Zeitler J, Gladden L. In vitro tomography and non-destructive imaging at depth of pharmaceutical solid dosage forms. Eur J Pharm Biopharm $2009 ; 71: 2-22$.

11. McGoverin CM, Rades T, Gordon KC. Recent pharmaceutical applications of raman and terahertz spectroscopies. J Pharm Sci 2008; $27: 27$.

12. Cook DJ, Chen JX, Morlino EA, Hochstrasser RM. Terahertz-field-induced second-harmonic generation measurements of liquid dynamics. Chem Physics Lett 1999; 309: 221-8.

13. Plusquellic DF, Siegrist K, Heilweil $\varepsilon$ J, Esenturk 0 . Applications of terahertz spectroscopy in biosystems. Chemphyschem $2007 ; 8: 2412-31$.

14. Le Boiteux S, Dell Fati N, Manek-Honninger I, Sarger L. THz-Time Domain Spectroscopy system for applications to radical detection. CR Acad Sci Paris $2001 ; 2: 983-8$.

15. Hindle F, Cuisset A, Bocquet R, Mouret G. Continuous-wave terahertz by photomixing: applications to gas phase pollutant detection and quantification. CR Physique $2008 ; 9: 262-75$.

16. Ho L, Muller R, Romer M, et al. Analysis of sustained-release tablet film coats using terahertz pulsed imaging. J Control Release 2007 ; 119: 253-61.

17. Product Brochure. Product brochure of Terahertz imaging system. Cambridge: TeraView Ltd, 2008.

18. Choi MK, Bettermann A, Van der Weide DW. Potential for detection of explosive and biological hazards with electronic terahertz systems. Philos Transact A Math Phys Eng Sci 2004 ; 362 : 337-49.

19. Fitzgerald AJ, Berry $\varepsilon$, Zinovev NN, et al. An introduction to medical imaging with coherent terahertz frequency radiation. Phys Med Biol $2002 ; 47:$ R67-84.

20. Siebert K, Löffler T, Quast $H$, et al. All-optoelectronic continuous wave THz imaging for biomedical applications. Phys Med Biol 2002 ; $47: 3743-8$.

21. Sim YC, Maeng I, Son JH. Frequency-dependent characteristics of terahertz radiation on the enamel and dentin of human tooth. Curr Appl Phys $2009 ; 9: 946-9$.

22. Ciesla CM, Armone DD, Pepper M. Biomedical applications of terahertz pulse imaging. SPIE $2000 ; 73-81$.

23. Wallace VP. Terahertz methods show promise for breast cancer. Terahertz Imaging $2006 ; 83-5$.

24. Wu $Q$, Zhang XC. Ultrafast electro-optic field sensors. Appl Phys Lett $1996 ; 68: 1604-6$.

25. Zeni 0 , Gallerano GP, Perrotta A, et al. Cytogenetic observations in human peripheral blood leukocytes following in vitro exposure to $\mathrm{THz}$ radiation: a pilot study. Health Phys 2007 ; 92 : 349-57.

26. Doria A, Gallerano GP, Giovenale $\varepsilon$, et al. THz radiation studies on biological systems at the ENEA FEL facility. Infrared Physics Technology $2004 ; 45: 339-47$.

27. Korenstein-Ilan A, Barbul A, Hasin P, et al. Terahertz radiation increases genomic instability in human lymphocytes. Radiat Res 2008 ; $170: 224-34$.

28. Redo Sanchez A, Xi Cheng Z. Terahertz science and technology trends. દદદ J Sel Top Quantum Electron 2008; $14: 260-9$.

29. Coutaz JL. Recent developments in terahertz optoelectronics. CR Physique $2008 ; 9: 127-9$. 